\section{INVESTIGATION}

Research reported in the $B D J$ in January (BDJ 2014; 216: 5) suggests that individual intake of 'free sugars' should be less than $5 \%$ of calories - around five teaspoons a day - to minimise the risk of dental cavities throughout life. ${ }^{1}$ Free sugars are those that are added to food as well as those naturally present in honey, syrups, fruit juices and fruit concentrates.

Previously, the World Health Organisation (WHO) had recommended that intake of free sugars should be less than $10 \%$ of total calorie intake.

Although fully aware of my sweet tooth and my tendency to look for something sweet to round off a meal - even if that is 'only a yoghurt' - I don't consider myself to have a diet high in sugar. I don't take sugar in tea or coffee, don't drink fizzy drinks, and try to use a straw when I drink fruit juice. Admittedly, I do have a number of fillings, but these days (after a decade in dental publishing), I am scrupulous about keeping my mouth clean and my dentist is happy to see me just once a year.

However, I wondered just how much sugar I consume in an average week, and if it is anywhere near the five teaspoon threshold. So, I decided to keep a food diary for one week, and then used a handy website (myfitnesspal.com) to tot up the approximate sugar quantities. By way of comparison, and for moral support, my colleague Lucy offered to do the same.

In the following sugar diaries, I have only included the food and drinks that contained sugar rather than everything we consumed. One teaspoon of sugar is $4 \mathrm{~g}$ so five teaspoons a day is $20 \mathrm{~g}$.

1. Moynihan P J, Kelly S A. Effect on caries of restricting sugars intake: systematic review to inform WHO guidelines. J Dent Res 2014; 93: 8-18.

Look out for further sugar features in future issues.

BY KATE QUINLAN

\title{
THE SUGAR DIARIES
}

KATE QUINLAN

\section{Monday}

Jordans Country Crisp

cereal with Flame Raisins

and added raspberries

33 g sugar

Satsuma $\mathbf{5}$ g sugar

Home-made sandwich $\mathbf{2} \mathbf{g}$ sugar

Cadbury's Creme Egg $\mathbf{2 6}$ g sugar

Slice of homemade cake $\mathbf{3 0 g}$ sugar

Glass red wine $\mathbf{2} \mathbf{g}$ sugar

Day total $=65 \mathrm{~g}$ sugar $=16$ teaspoons

\section{Shrove Tuesday}

Toast with Nutella $\mathbf{9}$ g sugar

Natural yoghurt with raspberry layer $\mathbf{5} \mathbf{g}$ sugar

One biscuit $7 \mathbf{g}$ sugar

One small apple $\mathbf{1 5} \mathbf{g}$ sugar

Homemade drop scones with various sweet

toppings $\mathbf{4 0} \mathbf{g}$ sugar

Glass red wine $2 \mathrm{~g}$ sugar

Day total $=78 \mathrm{~g}=19.5$ teaspoons

\section{Wednesday}

Homemade drop scones with lemon curd $\mathbf{5}$ g sugar Mini Cheddars $1.2 \mathrm{~g}$ sugar

Black Cherry Activia yoghurt $12 \mathrm{~g}$ sugar

Three chocolates $\mathbf{5}$ g sugar

Orange Club biscuit 12 g sugar (after giving blood!)

Raspberry ripple ice cream $\mathbf{2 0}$ g sugar

Glass red wine $\mathbf{2} \mathbf{g}$ sugar

Day total $=57.2 \mathrm{~g}=14.3$ teaspoons

\section{Thursday}

Homemade drop scones with Nutella $\mathbf{9} \mathbf{g}$ sugar Jordans Country Crisp cereal with Flame Raisins

32 g sugar

Vanilla Activia yoghurt 12 g sugar

Banana 12 g sugar

Raisins $15 \mathrm{~g}$ sugar

Caramelised onions (in tart) $10 \mathrm{~g}$ sugar

Glass red wine $\mathbf{2} \mathbf{g}$ sugar

Day total $=92 \mathrm{~g}=23$ teaspoons

\section{Friday}

Special K with Red Berries 9 g sugar

Banana 12 g sugar

Strawberry Activia yoghurt $12 \mathbf{g}$ sugar

Slice of chocolate birthday cake $\mathbf{2 6 . 8} \mathbf{g}$ sugar

Raspberry ripple ice cream $\mathbf{2 0}$ g sugar

Glass red wine $\mathbf{2} \mathbf{g}$ sugar

Day total $=81.8 \mathrm{~g}=20.4$ teaspoons

\section{Saturday}

Yoghurt with blackberries $15 \mathrm{~g}$ sugar

Homemade cake $10 \mathrm{~g}$ sugar

Caramelised onions (in tart) $10 \mathrm{~g}$ sugar

Day total $=35 \mathrm{~g}=8.7$ teaspoons

Sunday

Home-made cake $10 \mathrm{~g}$ sugar

Mini Magnum $12 \mathrm{~g}$ sugar

Roast vegetables $\mathbf{8} \mathbf{g}$ sugar

Red wine $6 \mathbf{g}$ sugar

Day total $=36 \mathrm{~g}=9$ teaspoons

DAILY AVERAGE = 15.8 teaspoons - over

three times the maximum amount of daily sugars suggested in the research.

Verdict: It looks like I should cut down on cake, ice cream, yoghurt - and probably red wine - to reduce my risk of future dental cavities.

\section{LUCY HEANEY}

\section{Monday}

Five teaspoons added to drinks $20 \mathrm{~g}$ sugar Berries added to porridge $\mathbf{2 1 . 4}$ g sugar Caramelised cashews and peanuts $11 \mathrm{~g}$ sugar

Baked beans $10.3 \mathrm{~g}$ sugar

White bread 1.5 sugar

Chocolate honeycomb bites $17 \mathrm{~g}$ sugar $500 \mathrm{ml}$ Coke $\mathbf{5 3} \mathbf{g}$ sugar

Day total $=146 \mathrm{~g}$ sugar $=36.5$ teaspoons

\section{Shrove Tuesday}

Four teaspoons added to drinks $16 \mathbf{g}$ sugar Apple chips added to porridge $13.9 \mathrm{~g}$ sugar Hoisin chicken $\mathbf{7 . 6}$ g sugar

Sour cream $6 \mathbf{g}$ sugar

Hot chocolate $24.1 \mathrm{~g}$ sugar

Tiffin biscuit cake $\mathbf{4 8 . 5} \mathbf{g}$ sugar

Pancakes with maple syrup $12 \mathrm{~g}$ sugar

$500 \mathrm{ml}$ Coke $\mathbf{5 3}$ g sugar

Day total $=181 \mathrm{~g}$ sugar $=45$ teaspoons

\section{Wednesday}

Two teaspoons added to drinks $\mathbf{8} \mathbf{g}$ sugar Apple chips added to porridge $13.9 \mathrm{~g}$ sugar Thai chicken soup $\mathbf{2 0} \mathbf{g}$ sugar Houmous and salad wrap $4.4 \mathrm{~g}$ sugar

Banana 12 g sugar

Fillet-o-fish 5 g sugar

Day total $=68.3 \mathrm{~g}$ sugar $=17$ teaspoons

Thursday

Two teaspoons added to drinks $\mathbf{8} \mathbf{g}$ sugar Berries added to porridge $\mathbf{2 1 . 4} \mathrm{g}$ sugar Lemon cheesecake $\mathbf{2 6 . 6} \mathbf{g}$ sugar

Prawn and chili dip snack $11.9 \mathrm{~g}$ sugar Tesco Finest Fish pie $\mathbf{6 . 8} \mathbf{g}$ sugar

Profiterole dessert $\mathbf{2 0} \mathrm{g}$ sugar

Day total $=99.9 \mathrm{~g}$ sugar $=24.9$ teaspoons

Friday

Pret a Manger bacon and egg roll $\mathbf{3 . 3}$ g sugar Three teaspoons added to drinks $12 \mathbf{g}$ sugar Chicken burger with mango and lime $\mathbf{6} \mathbf{g}$ sugar Graze snacks $51.6 \mathrm{~g}$ sugar

Day total $=72.9 \mathrm{~g}$ sugar $=18.2$ teaspoons

\section{Saturday}

Four teaspoons added to drinks $16 \mathbf{g}$ sugar White bread $\mathbf{5 . 1} \mathbf{g}$ sugar

Marinated prawns with chili dip $11.9 \mathrm{~g}$ sugar

Lemon trifle $\mathbf{4} \mathbf{g}$ sugar

Pomegranate juice $13 \mathrm{~g}$ sugar

Day total $=58.3 \mathrm{~g}$ sugar $=14.5$ teaspoons

\section{Sunday}

Three teaspoons added to drinks 12 g sugar White bread, brown sauce (with eggs) $15 \mathbf{g}$ sugar Tesco large sushi snack pack $\mathbf{9} \mathbf{g}$ sugar

Activia yoghurt $7.9 \mathrm{~g}$ sugar

Day total $=43.9 \mathrm{~g}$ sugar $=10.9$ teaspoons

DAILY AVERAGE = $\mathbf{2 3 . 8}$ teaspoons - over 4.5 times the maximum amount of daily sugars suggested in the research.

Verdict: Lucy has a bit of an addiction to sugary drinks, and cutting down on these would reduce her intake dramatically and also reduce her risk for future dental caries. 